\title{
Entropy generation in the flow system generated in between two parallel plates due to bivertical motion of the top plate
}

\author{
AHMET Z SAHIN and BEKIR S YILBAS \\ Mechanical Engineering Department, King Fahd University of Petroleum and \\ Minerals, Dhahran 31261, Saudi Arabia \\ e-mail: azsahin@kfupm.edu.sa
}

MS received 18 October 2007; revised 20 March 2009

\begin{abstract}
Thermodynamic irreversibility in the flow system provides information on the energy and power losses in the system. Minimization of entropy generation in the flow system enables for the parametric optimization of the system operation. In the present study, parallel plates, in between, filled with the fluid are considered. The fluid motion resulted from the bi-vertical compression of the top plate of the parallel plates is examined. The entropy generation rate in the flow system is formulated after considering the constant movement of the top plate, constant applied load, and the combination of the constant velocity and applied load to the top plate. The optimum operating conditions related to the fluid motion in between the parallel plates is determined through the entropy analysis. It is found that the combination of the constant velocity and the constant applied load resulted in the low entropy generation rate.
\end{abstract}

Keywords. Parallel plates; compression; fluid flow; entropy; second law analysis.

\section{Introduction}

The flow between the two parallel plates can be generated during the displacement of the one of the parallel plates. This situation is associated with the small displacement of the two parallel disks, in between, filled with the fluid, which are used in the metal forming industry. The power required for the plate motion depends on the speed of the plate and the force applied for the required displacement. However, the power required can be minimized through the adjustment of the process variables. In this case, the speed of the plate displacement, the force or the combination of speed and the applied force can be kept constant during the process. The entropy generation in the system provides insight into the power consumption through thermodynamic losses. Therefore, the entropy minimization provides power optimization for the plate motion in the parallel plate system filled with the fluid. Consequently, investigation of entropy generation during the displacement of one of the plates in parallel plate system filled with the fluid becomes essential.

Considerable research studies were carried out to examine entropy generation in parallel plates for various applications. The entropy generation minimization in parallel plates in relation to the counter-flow heat exchangers was carried out by Ordonez \& Bejan (2000). They 
showed that the design could be optimized in terms of the ratio of the two-channel spacing and the total heat transfer area between the two streams. The experimental study of flow transition in a Taylor-Couette system was carried out by Takeda (1999). He showed that the total energy occupation and the global entropy generation could be used to identify the transition behaviour of the velocity field quantitatively. The thermodynamic analysis of thermomechanical coupling in Couette flow was considered by Demirel (2000). He demonstrated the effects of the pressure gradient and the Brinkman number on the volumetric entropy generation rate and the irreversibility distribution ratio. The entropy generation rate during fluid flow between two parallel plas with moving bottom plate was examined by Erbay et al (2003). They indicated that the rate of entropy generation attained the highest values as the Reynolds number increased while the lower plate was in counter motion. The steady state boundary layer equations over a flat plate with a constant wall temperature condition were solved using the integral method by Esfahani \& Jafarian (2005). They observed that the variation of the total entropy generation was the same as the variation of the boundary layer thickness. A design analysis based on the entropy minimization of welded stiffened square plate loaded by biaxial compression was carried out by Farkas et al (2005). They showed that the entropy based unconstrained minimization provided the design solution similar to that obtained from Rosenbrock's Hill-Climb algorithm. The entropy generation over a flat plate with isothermal and constant heat flux boundary conditions was examined by Ratts \& Brown (2004). They determined the entropy generation rate for incompressible laminar flow for large and small Prandtl numbers. The second law analysis on a flat plate fin array under cross flow was conducted by Lin \& Lee (2000). They showed that using the minimum entropy concept, an optimal Reynolds number existed over wide operating conditions.

In the present study, two parallel plates filled with the fluid are considered and entropy generation in the flow system due to bi-vertical motion of the top plate is examined. The influence of the plate velocity and the force applied to the plate on the entropy generation rate in the flow system is formulated. The minimum entropy generation through the combination of constant applied force and constant velocity of the plate is also investigated.

\section{Analysis}

The velocity distribution in e squeeze film between two parallel circular disks as shown in figure 1 is given by (Denn 1998),

$$
\begin{aligned}
& v_{z}=-3 V(t)\left[\frac{z}{H(t)}\right]^{2}\left[1-\frac{2}{3} \frac{z}{H(t)}\right]=\phi(z) \\
& v_{r}=\frac{3 r z V(t)}{H^{2}(t)}\left[1-\frac{z}{H(t)}\right],
\end{aligned}
$$

where $V(t)=-\frac{d H}{d t}$.

The fluid flow is assumed to be isothermal, cylindrical axisymmetric and two dimensional. Furthermore, the axial velocity component $v_{z}$ is assumed to be a function of axial coordinate $\mathrm{z}$ only. The volumetric entropy generation rate in cylindrical coordinates is given by

$$
\dot{s}_{\text {gen }}^{\prime \prime \prime}=\frac{\mu}{T}\left\{2\left[\left(\frac{\partial v_{r}}{\partial r}\right)^{2}+\left(\frac{v_{r}}{r}\right)^{2}+\left(\frac{\partial v_{z}}{\partial z}\right)^{2}\right]+\left(\frac{\partial v_{z}}{\partial r}+\frac{\partial v_{r}}{\partial z}\right)^{2}\right\} .
$$




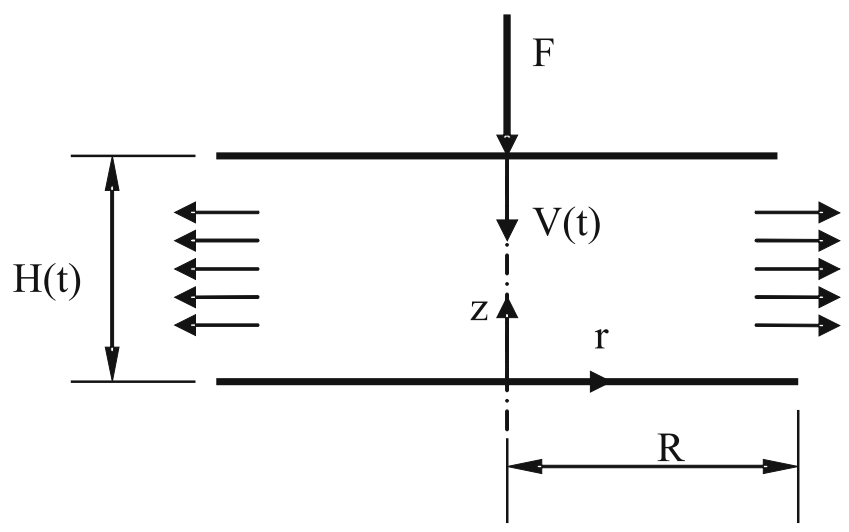

Figure 1. Schematic cross section of a squeeze film.

Since $v_{z}=\phi(z)$ the volumetric entropy generation rate simplifies to

$$
\dot{s}_{\text {gen }}^{\prime \prime \prime}=\frac{\mu}{T}\left\{2\left[\left(\frac{\partial v_{r}}{\partial r}\right)^{2}+\left(\frac{v_{r}}{r}\right)^{2}+\left(\frac{\partial v_{z}}{\partial z}\right)^{2}\right]+\left(\frac{\partial v_{r}}{\partial z}\right)^{2}\right\} .
$$

Using the velocity components given in equations (1) and (2) it can be shown that the volumetric entropy generation rate at time $t$ is

$$
\dot{s}_{\text {gen }}^{\prime \prime \prime}(t)=9 \frac{\mu}{T}\left(\frac{V}{R}\right)^{2}\left(\frac{R}{H}\right)^{6}\left[12\left(\frac{z}{R}\right)^{2}\left(\frac{H}{R}-\frac{z}{R}\right)^{2}+\left(\frac{r}{R}\right)^{2}\left(\frac{H}{R}-2 \frac{z}{R}\right)^{2}\right],
$$

where $V(t)=-\frac{d H}{d t}$.

The total (volume integrated) entropy generation rate becomes

$$
\dot{s}_{\text {gen }}(t)=\int_{z=0}^{H} \int_{r=0}^{R} \dot{s}_{\text {gen }}^{\prime \prime \prime}(2 \pi r) d r d z
$$

or

$$
\dot{s}_{\text {gen }}(t)=\frac{\mu}{T}\left(\frac{V}{R}\right)^{2}\left(\pi R^{2} H\right)\left(\frac{R}{H}\right)^{2}\left[\frac{18}{5}+\frac{3}{2}\left(\frac{R}{H}\right)^{2}\right],
$$

where $V(t)=-\frac{d H}{d t}$. The total entropy generation rate is a function of the velocity of the upper plate $V$ and the aspect ratio $H / R$ besides other constant properties as can be seen in equation (7).

\subsection{Case 1: Constant plate velocity}

In this case let the upper plate velocity be constant

$$
V(t)=V_{0}=\text { const. }
$$

Considering the problem geometry given in figure 1

$$
\frac{d H}{d t}=-V_{0} \text {. }
$$


The total entropy generation during the entire process of squeezing can be calculated by integrating equation (7) with time for the squeeze process duration from an initial spacing of $H_{o}$ to a final spacing of $H_{f}$

$$
s_{T}=\int_{0}^{t_{f}} \dot{s}_{\text {gen }}(t) d t=-\frac{1}{V_{0}} \int_{H_{0}}^{H_{f}} \dot{s}_{\text {gen }} d H .
$$

Carrying out the integration, it can be shown that the total entropy generation becomes

$$
s_{T}=\pi \frac{\mu}{T} R^{2} V_{0}\left\{\frac{18}{5} \ln \left(\frac{H_{0}}{H_{f}}\right)+\frac{3}{4}\left(\frac{R}{H_{0}}\right)^{2}\left[\left(\frac{H_{0}}{H_{f}}\right)^{2}-1\right]\right\}
$$

or in a dimensionless form it becomes

$$
\sigma_{V}=\frac{s_{T}(V=\text { const })}{\frac{\mu}{T} \frac{V_{0}}{H_{0}}\left(\pi R^{2} H_{0}\right)}=\frac{18}{5} \ln \left(\frac{H_{0} / R}{H_{f} / R}\right)+\frac{3}{4}\left(\frac{R}{H_{0}}\right)^{2}\left[\left(\frac{H_{0} / R}{H_{f} / R}\right)^{2}-1\right] .
$$

Equation (12) indicates that the total entropy generation increases as the final spacing $H_{f}$ decreases and goes to infinity as $H_{f}$ goes to zero. In fact, the required force to keep the upper plate velocity constant is inversely proportional to the cube of the plate spacing (Denn 1998)

$$
F=\frac{3 \pi \mu V R^{4}}{2 H^{3}} .
$$

Therefore the force also reaches infinity when the plate spacing reaches zero. This means that the upper place velocity can not be kept constant all the way to reduce the spacing to zero. Then, a possible way for the process is to keep the applied force constant in which case the plate velocity will not be constant and will decrease as the spacing decreases. This case is investigated in the following section.

\subsection{Case 2: Constant applied force}

In this case the force applied to the upper plate is assumed to be constant

$$
F=\frac{3 \pi \mu V R^{4}}{2 H^{3}}=\text { const. }
$$

Thus, the velocity of the upper plate is given to be

$$
V(t)=\frac{2 F}{3 \pi \mu R^{4}}[H(t)]^{3} .
$$

Substituting equation (15) into equation (7) we obtain

$$
\dot{s}_{\text {gen }}(t)=\frac{F^{2}}{\pi \mu T R}\left(\frac{H}{R}\right)^{3}\left[\frac{8}{5}\left(\frac{H}{R}\right)^{2}+\frac{2}{3}\right]
$$

in which

$$
\frac{d H(t)}{d t}=-V(t) .
$$


Equation (16) can be written in dimensionless form as

$$
\sigma_{\mathrm{gen}}(t)=\frac{\dot{s}_{\mathrm{gen}}(t)}{\frac{F^{2}}{\pi \mu T R}}=\left(\frac{H}{R}\right)^{3}\left[\frac{8}{5}\left(\frac{H}{R}\right)^{2}+\frac{2}{3}\right] .
$$

In this case, the total (volume integrated) entropy generation rate becomes a function of the aspect ratio $H(t) / R$ as given in equation (18).

The total entropy generation during the squeeze process in this case is

$$
s_{T}=\int_{0}^{t_{f}} \dot{s}_{\text {gen }}(t) d t=-\int_{H_{0}}^{H_{f}} \frac{1}{V} \dot{s}_{\text {gen }} d H .
$$

Using equation (17) and carrying out the integration in equation (19) the total entropy generation for the process is obtained as

$$
s_{T}=\frac{F R}{T}\left\{\frac{4}{5}\left[\left(\frac{H_{0}}{R}\right)^{3}-\left(\frac{H_{f}}{R}\right)^{3}\right]+\left(\frac{H_{0}}{R}-\frac{H_{f}}{R}\right)\right\} .
$$

The dimensionless form of the entropy generation in this case can be written as

$$
\sigma_{F}=\frac{s_{T}(F=\text { const })}{\frac{F R}{T}}=\frac{4}{5}\left[\left(\frac{H_{0}}{R}\right)^{3}-\left(\frac{H_{f}}{R}\right)^{3}\right]+\left(\frac{H_{0}}{R}-\frac{H_{f}}{R}\right) .
$$

As the final spacing is reduced to zero the dimensionless total entropy generation becomes

$$
\sigma_{F}\left(H_{f}=0\right)=\frac{4}{5}\left(\frac{H_{0}}{R}\right)^{3}+\frac{H_{0}}{R} .
$$

As the plate spacing is reduced to zero, the velocity also goes to zero as can be seen from the equation (15). This indicates that the time it takes to reach zero spacing becomes infinity. However, the entropy generation as indicated by equation (22) can be reduced to a finite value by keeping the applied force constant.

As the reducing the entropy generation is a primary interest, a third case can be considered to find a possible optimum operation in which the total entropy generation becomes minimum. This is treated in the next section where a two-step process is considered.

\subsection{Case 3: Two-step process}

In this case, we consider a two-step process: A constant velocity process followed by a constant force process. The constant velocity process takes place until a plate spacing of $\lambda H_{0}$ where $\lambda$ is the allocation parameter that can be varied between $0<\lambda<1$. This process is followed by a constant force process from the plate spacing of $\lambda H_{0}$ until zero spacing. In summary, this two-step process is

Step 1: $\left(H_{0} \Rightarrow \lambda H_{0}\right)$ : Constant Velocity $\left(V=V_{0}=\right.$ const $)$

Step 2: $\left(\lambda H_{0} \Rightarrow 0\right)$ : Constant Force $(F=$ const $)$. 
The total entropy generation for the constant velocity process (step 1) is

$$
s_{T}(V=\text { const })=-\frac{1}{V_{0}} \int_{H_{0}}^{\lambda H_{0}} s_{\text {gen }} d H .
$$

It follows from equation (11) and equation (23) that the total entropy generation becomes

$$
s_{T}(V=\text { const })=\pi \frac{\mu}{T} R^{2} V_{0}\left\{\frac{18}{5} \ln \left(\frac{1}{\lambda}\right)+\frac{3}{4}\left(\frac{R}{H_{0}}\right)^{2}\left[\left(\frac{1}{\lambda}\right)^{2}-1\right]\right\}
$$

or in dimensionless form

$$
\sigma_{V}=\frac{s_{T}(V=\text { const })}{\pi \frac{\mu}{T} R^{2} V_{0}}=\frac{18}{5} \ln \left(\frac{1}{\lambda}\right)+\frac{3}{4}\left(\frac{R}{H_{0}}\right)^{2}\left[\left(\frac{1}{\lambda}\right)^{2}-1\right] .
$$

For the constant force process (step 2) the force can be written as

$$
F=\frac{3 \pi \mu V R^{4}}{2 H^{3}}=\gamma \frac{3 \pi \mu V_{0} R^{4}}{2 H_{0}^{3}}
$$

where $\gamma$ is a parameter and $V_{0}$ is the constant velocity in step 1 and $H_{0}$ is the initial plate spacing.

The total entropy generation for this step can be obtained as

$$
s_{T}(F=\text { const })=-\frac{1}{V_{0}} \int_{\lambda H_{0}}^{0} s_{\text {gen }} d H .
$$

Using equation (20) the total entropy generation for this process becomes

$$
\begin{aligned}
s_{T}(F=\text { const }) & =\frac{F R}{T} \lambda\left(\frac{H_{0}}{R}\right)\left[\frac{4}{5} \lambda^{2}\left(\frac{H_{0}}{R}\right)^{2}+1\right] \\
& =\pi \frac{\mu}{T} V_{0} R^{2} \gamma \lambda\left[\frac{6}{5} \lambda^{2}+\frac{3}{2}\left(\frac{R}{H_{0}}\right)^{2}\right]
\end{aligned}
$$

or in dimensionless form

$$
\sigma_{F}=\frac{s_{T}(F=\text { const })}{\pi \frac{\mu}{T} V_{0} R^{2}}=\gamma \lambda\left[\frac{6}{5} \lambda^{2}+\frac{3}{2}\left(\frac{R}{H_{0}}\right)^{2}\right] .
$$

The overall total entropy generation for the two-step process is

$$
\begin{aligned}
\sigma_{T} & =\sigma_{V}+\sigma_{F} \\
& =\frac{18}{5} \ln \left(\frac{1}{\lambda}\right)+\frac{3}{4}\left(\frac{R}{H_{0}}\right)^{2}\left[\left(\frac{1}{\lambda}\right)^{2}-1\right]+\gamma \lambda\left[\frac{6}{5} \lambda^{2}+\frac{3}{2}\left(\frac{R}{H_{0}}\right)^{2}\right] .
\end{aligned}
$$




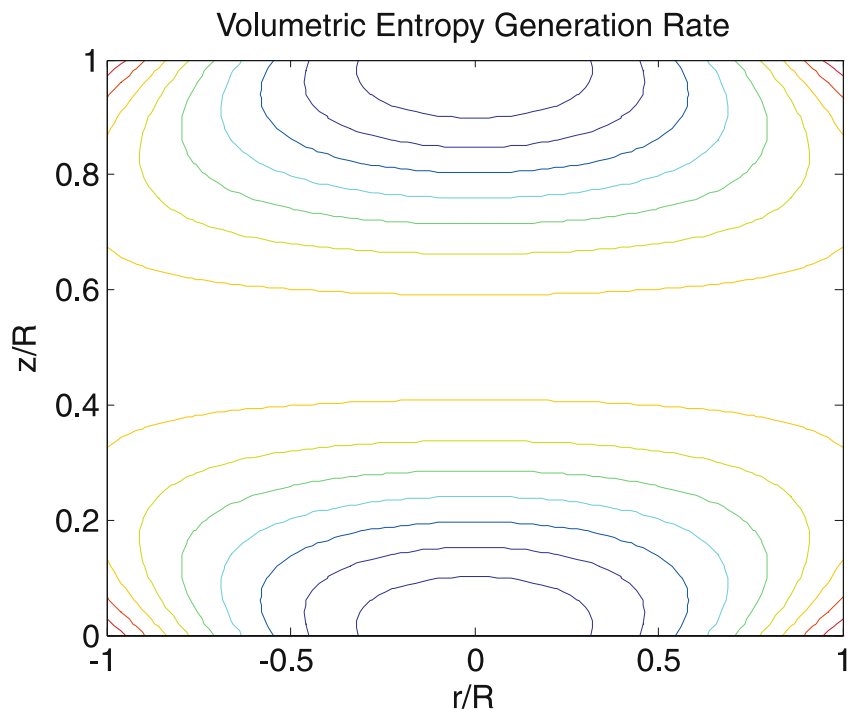

Figure 2. Dimensionless volumetric entropy generation rate $\dot{\sigma}_{\text {gen }}^{\prime \prime \prime}$ in squeeze film for $H=R$.

The optimum allocation parameter $\lambda_{\text {opt }}$ for the two-step process in which the total entropy generation becomes a maximum is obtained as

$$
\frac{d \sigma_{T}}{d \lambda}=0 \quad \Rightarrow \quad \lambda_{o p t}=\gamma^{-1 / 3}
$$

Thus the minimum entropy generation becomes

$$
\left(\sigma_{T}\right)_{\min }=\frac{6}{5} \ln (\gamma)+\frac{3}{4}\left(\frac{R}{H_{0}}\right)^{2}\left(3 \gamma^{\frac{2}{3}}-1\right)+\frac{6}{5} .
$$

\section{Results and discussion}

In the present work, the flow in the squeeze film is assumed to be isothermal. Therefore, the entropy generation rate depends on the fluid friction only. As can be seen from equation (5) the distribution of the volumetric entropy generation rate is a function of the viscosity of the fluid, upper plate velocity, temperature and the aspect ratio $H / R$. Figure 2 shows the distribution of the volumetric entropy generation rate throughout the film in dimensionless form $\dot{\sigma}_{\text {gen }}^{\prime \prime \prime}=\frac{\dot{s}_{\mathrm{gen}}^{\prime \prime \prime}(t)}{9 \frac{\mathrm{\mu}}{T}\left(\frac{V}{R}\right)^{2}}$ and for the case of $H=R$. The rate of volumetric entropy generation is symmetrical with respect to the mid-plane of the squeeze film. It increases away from the plates and in radial direction. The maximum volumetric entropy generation occurs near the plates close to the outer radius $r=R$. This is due to the high velocity gradients obtained in these locations. The volumetric entropy generation rate is constant in the mid-plane of the film and decreases to zero at the center of the disks.

Figure 3 shows the dimensionless volumetric entropy generation rate along the radius for different axial positions. The plate spacing $H$ is assumed to be equal to $R$. There is an extreme variation of volumetric entropy generation along the surface of the plates at $z=0$ from zero at the center to a maximum of 1 at the outer edge of the plates. At the center of the plates the 


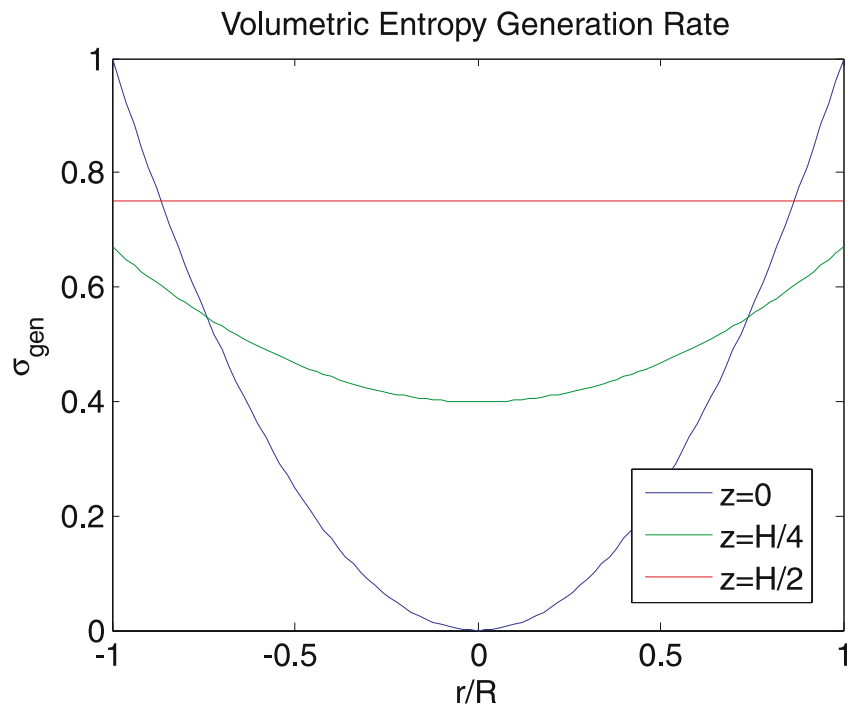

Figure 3. Dimensionless volumetric entropy generation rate along the radial direction for different axial locations and $H=R$.

velocity gradient is zero and reaches a maximum at the edge of the plates yielding maximum entropy generation. The dimensionless volumetric entropy generation rate at the mid-plane is uniform and equal to 0.75 . This is due to uniform distribution of the fluid velocity gradient in this plane.

Similarly the variation of the dimensionless entropy generation rate along the axial direction for different radial positions is given in figure 4. Maximum entropy generation occurs at the outer radius $r=R$. It varies from 0.75 at the mid-plane decreasing first and then increasing and reaching 1.0 at the plate surface. On the other hand, the dimensionless volumetric entropy generation rate along the centerline at $r=0$ varies from 0 at the plate surfaces and reaches to 0.75 at mid-plane of the squeeze film.

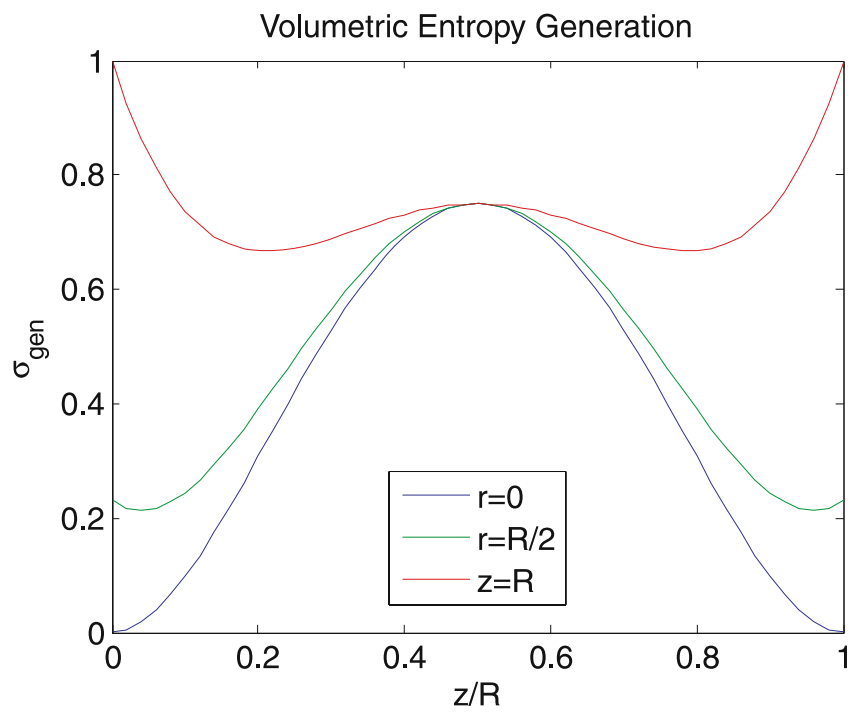

Figure 4. Dimensionless volumetric entropy generation rate along the axial direction for different radial locations and $H=R$. 


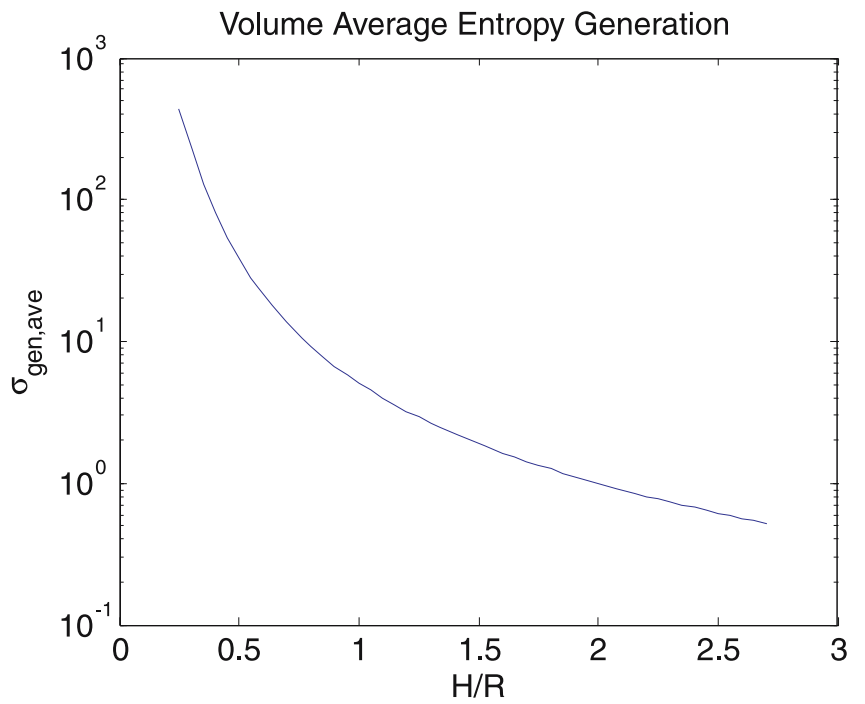

Figure 5. Variation of the dimensionless total entropy generation rate per unit volume in the squeeze film with dimensionless plate spacing.

Figure 5 shows the dimensionless total entropy generation rate per unit volume $\dot{\sigma}_{\text {gen,ave }}=$ $\frac{\dot{s}_{\mathrm{gen}}(t)}{\frac{\mu}{T}\left(\frac{V}{R}\right)^{2}\left(\pi R^{2} H\right)}$ as function of the dimensionless plate spacing from equation (7). As the plate spacing decreases the entropy generation increases considerably. This is due to the development of very high fluid velocity gradients. In the limit when the plate spacing approaches zero, the entropy generation reaches infinitely large values.

Figure 6 shows the dimensionless total entropy generation per unit volume of the squeeze film $\sigma_{V}$ as given in equation (12) when the film is squeezed with constant velocity from the initial spacing $H_{0}=R$ to a final spacing of $H_{f}$. As the final spacing $H_{f}$ decreases the total entropy generation increases considerably as mentioned above. Similarly, Figure 7 shows the dimensionless total entropy generation of the squeeze film $\sigma_{F}$ as given in equation (21) when

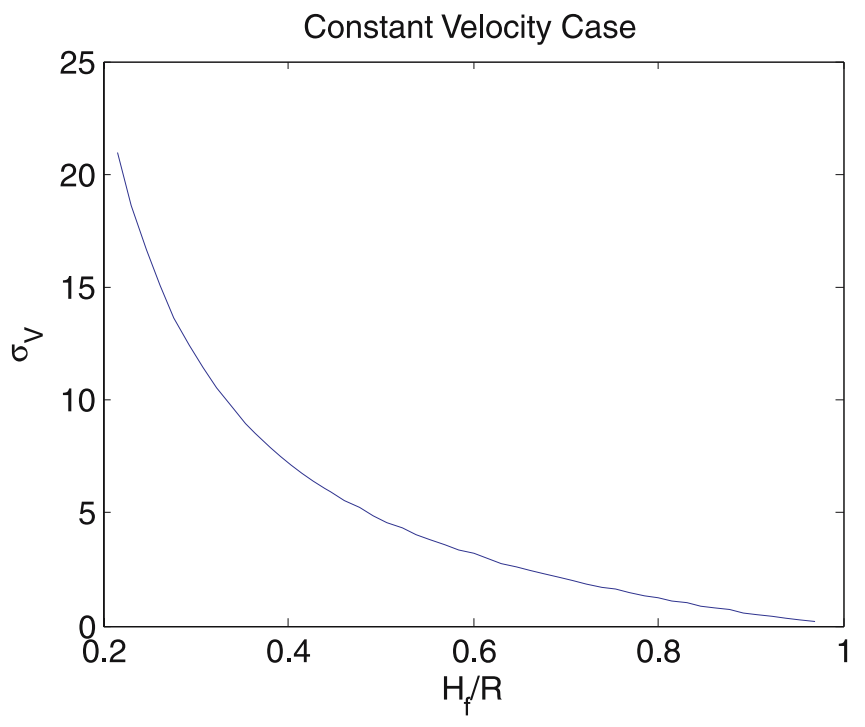

Figure 6. Variation of the dimensionless total entropy generation per unit volume in the squeeze film with dimensionless plate spacing for constant velocity case and $H_{0}=R$. 


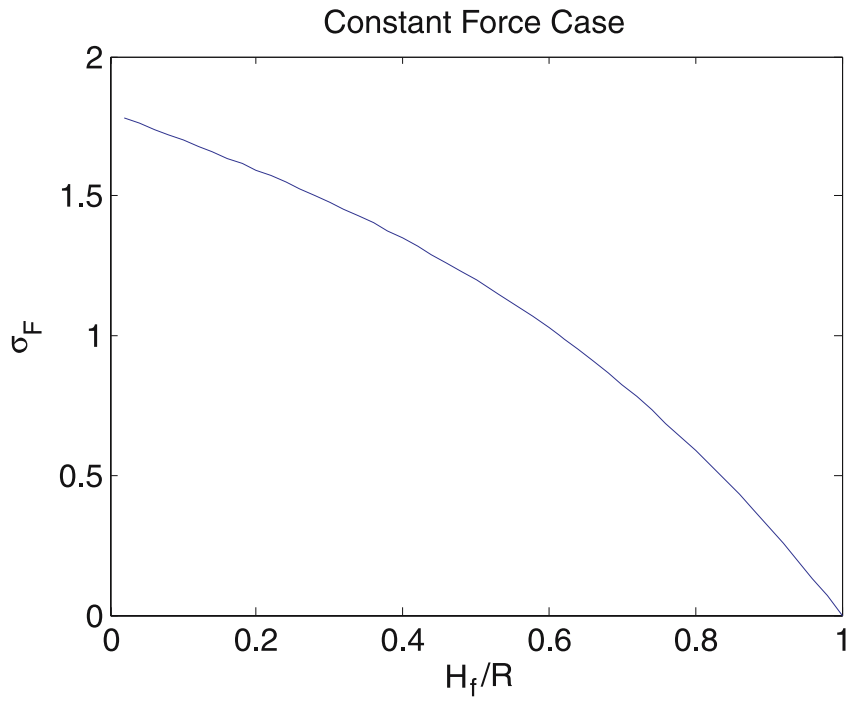

Figure 7. Variation of the dimensionless total entropy generation per unit volume in the squeeze film with dimensionless plate spacing for constant applied force case and $H_{0}=R$.

squeezed with a constant force from the initial spacing $H_{0}=R$ to a final spacing of $H_{f}$. The entropy generation increases as the final spacing is decreased but in this case it approaches to a constant value of 1.8 when the final spacing becomes zero. However, the process takes an infinitely long time.

Figure 8 shows the variation of the dimensionless total entropy generation in a two-step process for a force parameter of $\gamma=5$ as function of the allocation parameter $\lambda$. As the allocation parameter increases the total entropy generation for constant velocity case decreases but that for the constant force case increases. This indicates that the overall total entropy generation for the entire two-step process will have a minimum. The dimensionless overall total entropy generation during the two-step process is also shown in figure 8 . The minimum entropy generation occurs at about an allocation parameter of $\lambda=0.6$. The optimum allocation parameter

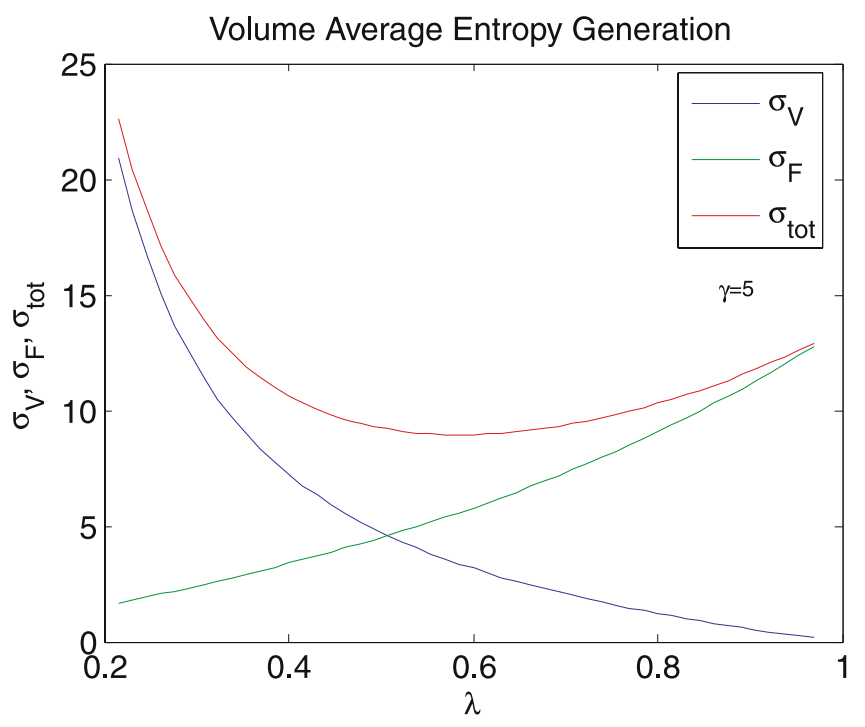

Figure 8. Variation of the dimensionless total entropy generation in a two-step squeeze process for a force parameter of $\gamma=5$ as function of the allocation parameter. 


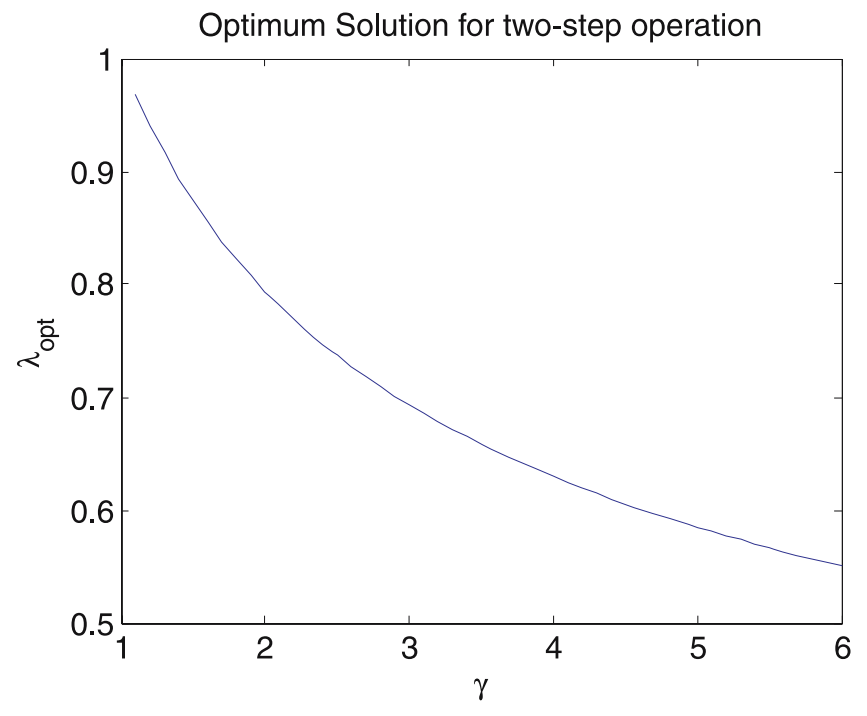

Figure 9. Variation of the optimum allocation parameter with force coefficient in two-step squeeze process.

depends on the force coefficient. The variation of the optimum allocation parameter with force coefficient in the two-step process is shown in figure 9. The optimum allocation parameter decreases as the force coefficient increases.

Figure 10 shows the dimensionless minimum total entropy generation as function of force coefficient and initial plate spacing as given in equation (32). As the initial plate spacing increases the total entropy generation increases. This increase is considerable especially for the low pressure coefficients. As the pressure coefficient increases the total entropy generation decreases. This corresponds to the application of small force during the second step and in turn low fluid flow velocity gradients during the process.

Minimum entropy generation for two-step operation

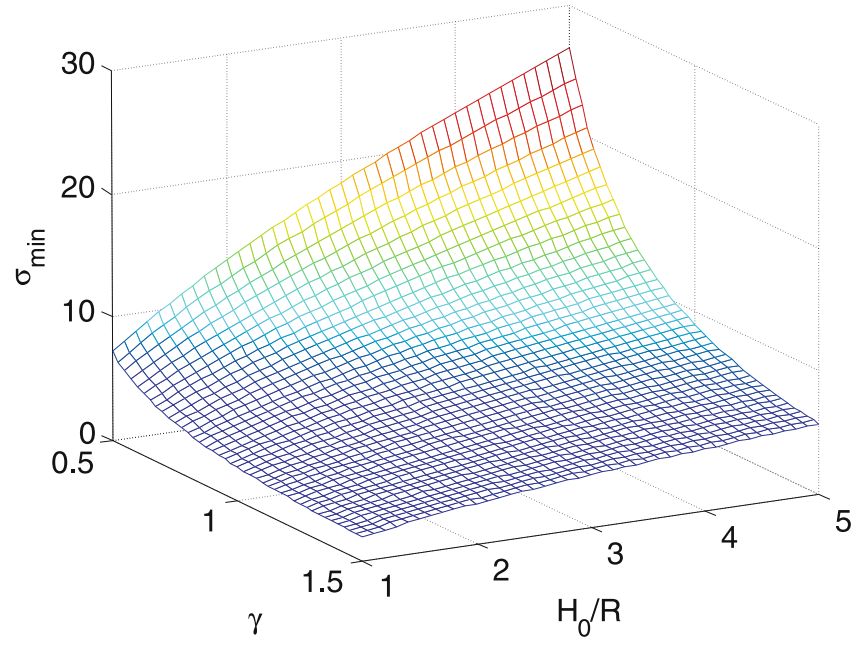

Figure 10. The optimum dimensionless total entropy generation in a two-step process as a function of pressure coefficient and initial plate spacing. 


\section{Conclusions}

Entropy generation rate in the flow filed developed between the parallel plates is considered and the effect of the bi-vertical motion of the top plate on the entropy generation rate is examined. The upper plate is displaced bi-vertically towards the bottom plate under the constant velocity, or the constant applied force, or the two-steps of first at the constant velocity and later at the constant force. It is found that increasing constant velocity lowers the entropy generation. This situation is also true for increasing constant force. However, the decay of entropy with the constant velocity is in the exponential form while it is parabolic form for the constant force case. Moreover, the combination of two-steps (first the constant velocity and later the constant force) results in the minimum entropy generation in the flow system. However, the minimum entropy occurs at specific displacement value; in which case the constant velocity operation should continue to reach a particular gab height of the plates prior to the constant applied force operation. Consequently, two-steps operation provides the minimum entropy generation and minimum power loss in the working fluid between the two plates.

The authors acknowledge the support provided by King Fahd University of Petroleum and Minerals for this work.

\section{References}

Demirel Y 2000 Thermodynamic analysis of thermomechanical coupling in Couette flow. Int. J. Heat and Mass Transfer 43(22): 4205-4212

Denn M 1998 Process Fluid Mechanics, (New York: Prentice Hall)

Erbay L B, Ercan M S, Sulus B, Yalcin M M 2003 Entropy generation during fluid flow between two parallel plates with moving bottom plate. Entropy 5(5)

Esfahani J A, Jafarian M M 2005 Entropy generation analysis of a flat plate boundary layer with various solution methods. Scientia Iranica 12(2): 233-240

Farkas J, Simoes L M C V, Jamai K 2005 Minimum cost design of a welded stiffened square plate loaded by biaxial compression. Structural and Multidisciplinary Optimization 29(4): 298-303

Lin W W, Lee D J 2000 Second-law analysis on a flat plate fin array under crossflow. International Communications in Heat and Mass Transfer 27(2): 179-190

Ordonez J C, Bejan A 2000 Entropy generation minimization in parallel-plates counterflow heat exchangers. Int. J. Energy Res. 24(10): 843-864

Ratts E B, Brown J S 2004 Laminar entropy generation over a flat plate with isothermal and constant heat flux boundary conditions using the von Karman integral method, In: Proceedings of the ASME Heat Tranfer/Fluids Engineering Summer Conference, HT/FED 2004, Charlotte, NC, USA 1: 61-69

Takeda Y 1999 Quasi-periodic state and transition to turbulence in a rotating Couette system. J. Fluid Mechanics 389: 81-99 